\title{
Attitudes towards risk-reducing early salpingectomy with delayed oophorectomy for ovarian cancer prevention: a cohort study
}

\author{
F Gaba, ${ }^{\text {a,b }}$ (D) O Blyuss, ${ }^{c, d, e}$ D Chandrasekaran,,${ }^{\text {a,b }}$ M Osman, ${ }^{a}$ S Goyal, ${ }^{a}$ C Gan, ${ }^{f}$ L Izatt, ${ }^{9}$ V Tripathi, ${ }^{g}$

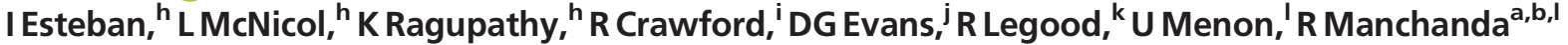 \\ ${ }^{a}$ Wolfson Institute of Preventive Medicine, Cancer Research UK, Barts Centre, Queen Mary University of London, Charterhouse Square, \\ London, UK ${ }^{\mathrm{b}}$ Department of Gynaecological Oncology, St Bartholomew's Hospital, Barts Health NHS Trust, London, UK ${ }^{\mathrm{c}}$ School of \\ Physics, Astronomy and Mathematics, University of Hertfordshire, Hatfield, UK ${ }^{\mathrm{d}}$ Department of Paediatrics and Paediatric Infectious \\ Diseases, Sechenov First Moscow State Medical University, Moscow, Russia ${ }^{e}$ Department of Applied Mathematics, Lobachevsky State \\ University of Nizhny Novgorod, Nizhny Novgorod, Russia ${ }^{\mathrm{f}}$ Department of Gynaecological Oncology, Nottingham University Hospitals NHS \\ Trust, Nottingham, UK ${ }^{\mathrm{g}}$ Department of Clinical Genetics, Guy's Hospital, London, UK ${ }^{\mathrm{h}}$ Ninewells Hospital, NHS Tayside, Dundee, UK \\ ${ }^{i}$ Department of Gynaecological Oncology, Addenbrooke's Hospital, Cambridge University Hospitals NHS Foundation Trust, Cambridge, UK \\ ${ }^{j}$ Manchester Centre for Genomic Medicine, MAHSC, Division of Evolution and Genomic Sciences, University of Manchester, Manchester, UK \\ ${ }^{\mathrm{k}}$ Department of Health Services Research and Policy, London School of Hygiene and Tropical Medicine, London, UK ${ }^{1}$ MRC Clinical Trials \\ Unit, University College London, London, UK \\ Correspondence: R Manchanda, Wolfson Institute of Preventive Medicine, Barts CRUK Cancer Centre, Queen Mary University of London, \\ Charterhouse Square, London EC1M 6BQ, UK. Email: r.manchanda@qmul.ac.uk
}

Accepted 13 July 2020

Objective To determine risk-reducing early salpingectomy and delayed oophorectomy (RRESDO) acceptability and effect of surgical prevention on menopausal sequelae/satisfaction/regret in women at increased ovarian cancer (OC) risk.

Design Multicentre, cohort, questionnaire study (IRSCTN:12310993).

Setting United Kingdom (UK).

Population UK women without OC $\geq 18$ years, at increased OC risk, with/without previous RRSO, ascertained through specialist familial cancer/genetic clinics and BRCA support groups.

Methods Participants completed a 39-item questionnaire. Baseline characteristics were described using descriptive statistics. Logistic/ linear regression models analysed the impact of variables on RRESDO acceptability and health outcomes.

Main outcomes RRESDO acceptability, menopausal sequelae, satisfaction/regret.

Results In all, 346 of 683 participants underwent risk-reducing salpingo-oophorectomy (RRSO). Of premenopausal women who had not undergone RRSO, 69.1\% (181/262) found it acceptable to participate in a research study offering RRESDO. Premenopausal women concerned about sexual dysfunction were more likely to find RRESDO acceptable (odds ratio $[\mathrm{OR}]=2.9,95 \%$ CI $1.2-7.7$,
$P=0.025)$. Women experiencing sexual dysfunction after premenopausal RRSO were more likely to find RRESDO acceptable in retrospect $(\mathrm{OR}=5.3,95 \% \mathrm{CI} 1.2-27.5, P<0.031)$. In all, $88.8 \%(143 / 161)$ premenopausal and $95.2 \%(80 / 84)$ postmenopausal women who underwent RRSO, respectively, were satisfied with their decision, whereas $9.4 \%(15 / 160)$ premenopausal and $1.2 \%$ (1/81) postmenopausal women who underwent RRSO regretted their decision. HRT uptake in premenopausal individuals without breast cancer (BC) was $74.1 \%$ (80/108). HRT use did not significantly affect satisfaction/regret levels but did reduce symptoms of vaginal dryness $(\mathrm{OR}=0.4$, 95\% CI $0.2-0.9, P=0.025$ ).

Conclusion Data show high RRESDO acceptability, particularly in women concerned about sexual dysfunction. Although RRSO satisfaction remains high, regret rates are much higher for premenopausal women than for postmenopausal women. HRT use following premenopausal RRSO does not increase satisfaction but does reduce vaginal dryness.

Keywords Acceptability, $B R C A$, ovarian cancer, risk-reducing early salpingectomy with delayed oophorectomy.

Tweetable abstract RRESDO has high acceptability among premenopausal women at increased ovarian cancer risk, particularly those concerned about sexual dysfunction.

Please cite this paper as: Gaba F, Blyuss O, Chandrasekaran D, Osman M, Goyal S, Gan C, Izatt L, Tripathi V, Esteban I, McNicol L, Ragupathy K, Crawford R, Evans DG, Legood R, Menon U, Manchanda R. Attitudes towards risk-reducing early salpingectomy with delayed oophorectomy for ovarian cancer prevention: a cohort study. BJOG 2020; https://doi.org/10.1111/1471-0528.16424. 


\section{Introduction}

Ovarian cancer (OC) is the leading cause of death from gynaecological malignancies in the UK. ${ }^{1}$ An effective OCscreening programme/strategy is not currently clinically available and 10-year survival rates remain poor at $\sim 30 \%$. Familial cancers are responsible for $\sim 10-20 \%$ of OC and $B R C A 1 / B R C A 2$ mutations account for most of the known hereditary OC risk. BRCA1/BRCA2 carriers have a $17-44 \%$ OC risk and $65-72 \%$ breast cancer (BC) risk. ${ }^{2-5}$ RAD51C/ RAD51D/BRIP1 are newer moderate-penetrance OC genes, with lifetime OC risks $\sim 6-13 \% .^{6-10}$

Risk-reducing salpingo-oophorectomy (RRSO) is the most effective method of OC prevention. Traditionally, women at $\geq 10 \%$ lifetime OC risk were deemed high risk and offered risk-management/surgical prevention. Calls for redefining the threshold for surgical prevention have suggested a $4-5 \%$ lifetime OC risk, ${ }^{11}$ as the level demonstrating clinical utility, ${ }^{12,13}$ thus enabling intermediate-risk women to access surgical prevention. This includes those with moderate-penetrance genetic mutations and mutationnegative women with a strong OC family history. In $B R C A$-women, RRSO reduces OC risk by $79-96 \% .^{14-18}$ Although initial data suggested that premenopausal RRSO reduced primary $\mathrm{BC}$ risk by half, ${ }^{16,19,20}$ recent papers have questioned this. $^{21,22}$ In terms of mortality, RRSO reduces all-cause $\quad(\mathrm{HR}=0.40, \quad 95 \% \quad \mathrm{CI} \quad 0.26-0.61), \quad$ BC-specific $(\mathrm{HR}=0.44, \quad 95 \% \quad \mathrm{CI} \quad 0.26-0.76) \quad$ and $\quad$ OC-specific $(\mathrm{HR}=0.25, \quad 95 \%$ CI $0.08-0.75)$ mortality. ${ }^{20}$ However, RRSO has disadvantages, including major complication rates of $1.5-5 \%{ }^{14,23}$ In premenopausal women, RRSO leads to surgical menopause and infertility. Premature surgical menopause has potential detrimental health sequelae including an increased risk of heart disease, osteoporosis, vasomotor symptoms, neurocognitive decline and sexual dysfunction, especially in women who do not or are unable to use hormone-replacement therapy (HRT). ${ }^{24-28}$ Consequently, some women choose to delay surgery until after menopause, risking a 6- to 16-year mean period of much higher risks of, in particular, high-grade serous OC, especially with BRCA1.

With increasing evidence and acceptability of the central role of the fallopian tube in the aetiopathogeneisis of epithelial OC, risk-reducing early salpingectomy and delayed oophorectomy (RRESDO) has been proposed as a two-stage surgical alternative to RRSO. RRESDO offers some level of risk reduction to women who decline/wish to delay RRSO while conserving ovarian function and avoiding detrimental consequences of premature menopause. ${ }^{29-31}$ However, prospective outcome data for RRESDO are lacking. The precise level of OC risk reduction and long-term consequences of ovarian function are unknown. Concerns have been raised regarding the potential attrition from delayed oophorectomy. Therefore, RRESDO is currently recommended in the context of a clinical trial and $80 \%$ of UK clinicians support this. ${ }^{32}$ Trials are currently underway in the UK (PROTECTOR; ISRCTN25173360), ${ }^{33}$ the Netherlands (TUBA; NCT02321228) ${ }^{34}$ and USA (WISP, NCT02760849). ${ }^{35}$

There is a paucity of international data and a lack of UK data on RRESDO acceptability among high-risk women. A US survey showed that $34.3 \%$ (70/204) BRCA pathogenicvariant carriers were interested in participating in a study offering RRESDO. ${ }^{36}$ A Dutch qualitative study investigating barriers and facilitators to RRESDO among BRCA-carriers found seriousness of OC, family history $(\mathrm{FH})$, previous $\mathrm{BC}$, uncertainty about the effect of and ease of the decision to undergo RRSO to be barriers. ${ }^{37}$ The main facilitator was longer maintenance of ovarian function to delay negative effects of premature menopause. ${ }^{37}$

We present data from a multicentre UK survey in women at increased OC risk, concerning: (1) acceptability of RRESDO, (2) menopausal sequelae following RRSO and (3) satisfaction/regret following RRSO.

\section{Methods}

\section{Design}

The present study was a multicentre, cohort, questionnaire study (ISRCTN:12310993). Inclusion criteria were: UK women aged $\geq 18$ years, at increased OC risk either due to pathogenic variants in an OC gene (BRCA1/BRCA2/ $R A D 51 C / R A D 51 D / B R I P 1)$ or strong $\mathrm{FH}$ of $\mathrm{OC}$ or $\mathrm{BC}+$ OC. A strong $\mathrm{FH}$ was defined as $\geq 2$ first-degree relatives with OC in BRCA1/BRCA2-negative or untested women. Exclusion criteria were: non-UK residents or women with a personal history of OC. Women with and without previous RRSO were invited.

\section{Participants}

Participants were selected via six NHS specialist familial cancer or genetic clinics (Manchester/Cambridge/BartsLondon/University-College-London/Guys-London/Dundee) and a patient support group for $B R C A$-carriers (BRCAUmbrella).

\section{Recruitment}

All participants provided written consent after review of a detailed participant information-sheet (PIS) along with the option of completing a paper/web-based questionnaire. The PIS (Appendix S1) provided information on current OCprophylactic surgery available on the NHS (RRSO) and the proposed two-stage surgical alternative (RRESDO), study aims, logistics of participation and contact details of 
charities/support groups providing further independent information/support.

The 39-item questionnaire collected socio-demographic, surgical/menstrual/FH and health consequences of premature menopause influencing decisions to undergo/delay/ decline premenopausal RRSO (Likert scale) data for all participants. Individuals were instructed to complete different sections of the questionnaire depending on whether they had undergone RRSO. Questionnaire items for women who had not undergone RRSO covered: benefits and limitations of RRESDO impacting acceptability of procedure (Likert scale); putative acceptability of undergoing RRESDO ('yes, no, not sure' options), acceptability of participating in a research study offering RRESDO (Likert scale); anticipated timing of future surgery (Likert scale). Questionnaire items for women who had undergone RRSO covered: retrospective acceptability (Likert scale) of undergoing RRESDO had it been available (item only for women who had undergone premenopausal RRSO); HRT use ('yes/ no'); menopause sequelae (Likert scale) following RRSO; satisfaction and regret (Likert scale) following RRSO. Respondents could recheck all answers and an optional free-text box was provided for further comments.

\section{Questionnaire development}

The 39-item questionnaire (Appendix S2) was developed in several stages. An initial draft was developed following a literature review. Each question was systematically discussed and debated. These were subsequently reviewed by senior clinicians in the fields of gynaecological precision medicine and cancer prevention, and gynaecological oncology. The clinicians gave each item a relevance score from 1 (least relevant) to 4 (most relevant) based on their knowledge and experience. They were also asked to identify any additional questions which they considered important and might be missing. A second consensus meeting was held to review responses to the initial questionnaire, delete lowrelevance items and optimise questionnaire length and facilitate compliance. A second pilot of the survey was carried out for readability, ease-of-use and layout. These processes helped ensure content and face optimisation. The final version was further reviewed/commented on, resulting in further rationalisation to a 39-item questionnaire. For questions pertaining to satisfaction/regret with decision making, the validated five-item decision regret scale $\mathrm{s}^{38,39}$ was used as well as two additional items developed by the panel of clinical experts exploring the impact of familial wishes on decision making.

\section{Statistical analysis}

Descriptive statistics were used for baseline characteristics as well as satisfaction/regret questions. Multiple logistic regression was used to model the effect of variables on the acceptability of undergoing RRESDO (putative acceptability in premenopausal women who had not undergone OC-prevention surgery; retrospective acceptability of RRESDO in women who had undergone premenopausal RRSO), acceptability of taking part in a research study offering RRESDO (premenopausal women not having undergone OC-prevention surgery) and effect of HRT uptake on menopausal sequelae in women who had undergone premenopausal RRSO. Multiple linear regression was used to model the effect of menopausal sequelae on satisfaction/regret following premenopausal RRSO, and association of HRT use versus non-use was also explored. Multiple analyses were adjusted for marital status, ethnicity, education, income, $\mathrm{FH}$ of OC/BC, risk-reducing mastectomy (RRM) and personal history of BC. Wilcoxon's rank-sum test and Fisher's exact test were using to test the hypothesis about differences in means and proportions, respectively. Two-sided $P$ values are reported for all statistical tests. Statistical analysis used R version 3.5.1 (Lucent Technologies, Murray Hill, NJ, USA).

\section{Patient and public involvement (PPI)}

The RRESDO study team undertook extensive stakeholder engagement before study commencement. Groups involved included healthcare professionals and BRCA support groups. This was essential to ensure stakeholder management, increase engagement and awareness and facilitate development and delivery of study. These groups provided input to the Patient Information Sheet and also served as an independent point of contact for more information on the study. Support groups helped increase study awareness through their websites/newsletters. They will also be involved in dissemination of study findings following publication.

\section{Core outcome sets}

There are no core outcome sets for surgical prevention at present.

\section{Results}

Between October 2017 and June 2019, 773 individuals completed the paper/online-questionnaire. Of these, 90 were excluded because they did not meet the eligibility criteria. The remaining 683 individuals were included in the analysis. Table 1 summarises baseline cohort characteristics. In all, 337/683 (49.3\%) respondents had not undergone RRSO and 346/683 (50.7\%) had. Women who had not undergone RRSO were significantly younger than women who had (38.3 versus 51.5 years, $P \leq 0.001$ ).

Table 2 summarises RRESDO acceptability. Among premenopausal women who had not undergone RRSO, the overall RRESDO acceptability ('yes') was 55.3\% (145/262) and the overall unacceptability ('no') was $20.2 \%$ (53/262); 
Gaba et al.

Table 1. Baseline characteristics of cohort

\begin{tabular}{|c|c|c|c|}
\hline & $\begin{array}{c}\text { No RRSO } \\
n=337,49.3 \%\end{array}$ & $\begin{array}{c}\text { RRSO } \\
n=346,50.7 \%\end{array}$ & $P$-value \\
\hline Mean age in years (SD, range) & $38.25(10.23,19-81)$ & $51.53(9.56,26-81)$ & $<0.001$ \\
\hline Mean parity (SD, range) & $1.92(0.83,1-6)$ & $2.18(0.86,1-7)$ & $<0.001$ \\
\hline Nulliparous & $217 / 336,64.6$ & $286 / 343,83.4$ & $<0.001$ \\
\hline Premenopausal/premenopausal at time of RRSO & $262 / 335,78.2$ & $164 / 248,66.1$ & 0.001 \\
\hline Postmenopausal/postmenopausal at time of RRSO & $73 / 335,21.8$ & $84 / 248,33.9$ & 0.001 \\
\hline Family complete & 194/331, 58.6 & $318 / 334,95.2$ & $<0.001$ \\
\hline \multicolumn{4}{|l|}{ High risk of $O C$} \\
\hline BRCA1 carrier & 157/337, 46.6 & $162 / 346,46.8$ & 1 \\
\hline$B R C A 2$-carrier & $171 / 337,50.7$ & $161 / 346,46.5$ & 0.284 \\
\hline$B R C A 1$ - and $B R C A 2$-carrier & $5 / 337,1.5$ & $7 / 346,2.0$ & 0.773 \\
\hline \multicolumn{4}{|l|}{ Intermediate risk of $\mathrm{OC}$} \\
\hline RAD51C-carrier & $0 / 337,0.0$ & $1 / 346,0.3$ & \\
\hline BRIP1-carrier & $3 / 337,0.9$ & $1 / 346,0.3$ & 0.367 \\
\hline$B R C A 1 / B R C A 2$-negative but strong $\mathrm{FH}$ of $\mathrm{OC}$ & $1 / 337,0.3$ & $2 / 346,0.6$ & 1 \\
\hline$B R C A 1 / B R C A 2$ untested but strong $\mathrm{FH}$ of $\mathrm{OC}$ & $1 / 337,0.3$ & $3 / 346,0.9$ & 0.624 \\
\hline Unsure of genetic test result & $4 / 337,1.2$ & $6 / 346,1.7$ & 0.752 \\
\hline Personal history of $B C$ & $77 / 337,22.8$ & $160 / 346,46.2$ & $<0.001$ \\
\hline Personal history of other cancers & $6 / 337,1.8$ & $9 / 346,2.6$ & 0.604 \\
\hline Therapeutic mastectomy & 20/337, 5.9 & $47 / 346,13.6$ & 0.001 \\
\hline Risk-reducing mastectomy & 70/337, 20.8 & $143 / 346,41.3$ & $<0.001$ \\
\hline Breast surgery for benign breast pathology & $0 / 337,0.0$ & $4 / 346,1.2$ & \\
\hline Cosmetic breast surgery & $2 / 337,0.6$ & $1 / 346,0.3$ & 0.620 \\
\hline \multicolumn{4}{|l|}{ Marital status } \\
\hline Single, divorced, separated, widowed & $72 / 332,21.7$ & $36 / 341,10.6$ & $<0.001$ \\
\hline Married, cohabiting/living with partner & $260 / 332,78.3$ & $305 / 341,89.4$ & $<0.001$ \\
\hline \multicolumn{4}{|l|}{ Ethnicity } \\
\hline Caucasian & $301 / 334,90.1$ & $300 / 341,88.0$ & 0.391 \\
\hline Non-caucasian & $33 / 334,9.9$ & $41 / 341,12.0$ & 0.391 \\
\hline \multicolumn{4}{|l|}{ Education } \\
\hline PhD, Masters, Bachelor's degree & $199 / 329,60.5$ & $141 / 336,42.0$ & $<0.001$ \\
\hline $\begin{array}{l}\text { NVQ4, A-level/NVQ3, NVQ1/NVQ2, GCSE/O-level/CSE, no formal qualification } \\
\text { Total household income }\end{array}$ & $130 / 329,39.5$ & $195 / 336,58.0$ & $<0.001$ \\
\hline$\geq £ 30000$ & $236 / 325,72.6$ & 208/312, 66.7 & 0.121 \\
\hline$<£ 30000$ & $89 / 325,27.4$ & $104 / 312,33.3$ & 0.121 \\
\hline
\end{tabular}

BC, breast cancer; FH, family history; OC, ovarian cancer; RRSO, risk-reducing salpingo-oophorectomy.

$90 / 773$ participants were excluded because they were at population-level risk of ovarian cancer. 683 participants eligible for analysis.

24.4\% (64/262) were 'not sure'. When premenopausal women who had not undergone RRSO were asked whether they would consider taking part in a research study offering RRESDO, overall acceptability (those who responded 'probably, maybe') was $69.1 \%(181 / 262)$ and $30.9 \%(81 / 262)$ found it unacceptable ('probably not, definitely not'). Table 2 provides further details. Multiple logistic-regression model outputs showing the association of covariates with acceptability of undergoing RRESDO ('yes' versus 'no') among premenopausal women who have not undergone RRSO are given in Table 3. Genetic-mutation type or carrier-status/ $\mathrm{FH} /$ knowledge of tubal origin/future OC-prevention surgery plans/childbearing/ethnicity/education/income did not statistically significantly affect RRESDO acceptability. However, premenopausal women who were more concerned about sexual dysfunction were three times more likely to find undergoing RRESDO acceptable than were those less concerned about sexual dysfunction $(\mathrm{OR}=2.9,95 \%$ CI 1.2-7.6, $P=0.025)$. When considering potential benefits, individuals wanting to delay hot flushes $(\mathrm{OR}=5.0,95 \%$ CI 1.2-21.2, $P=0.025)$ were five times more likely to find RRESDO acceptable. When considering acceptability ('yes' versus 'no') of potential limitations of RRESDO, women who found the risks of undergoing two surgeries (OR $=444.1,95 \%$ CI $28-$ 22815, $\quad P \leq 0.001$ ), interval-monitoring (CA125/USS) between surgeries $\quad(\mathrm{OR}=59.0, \quad 95 \% \quad$ CI $4.2-1548.7$, $P=0.006$ ), uncertainty around level of OC risk reduction with RRES (OR $=14.6$, 95\% CI 1.9-160.6, $P=0.015)$, and 
developing an interval OC between the two surgeries (OR $=9.6,95 \%$ CI 1.4-93.7, $P=0.032)$ as acceptable, were more likely to find undergoing RRESDO acceptable. The wide confidence intervals reflect having too few responses from premenopausal women who have not undergone RRSO for certain questionnaire items (acceptability of undergoing two surgeries/interval monitoring/uncertain OC risk reduction/developing interval OC). Multiple logistic-regression model outputs showing association of covariates with acceptability of undergoing RRESDO ('not sure' versus 'no') are given in Table S1. Premenopausal women who had not undergone RRSO and who responded 'not sure' were more likely than women who responded 'no' to find the two-stage aspect of RRESDO acceptable (OR $=6.7,95 \%$ CI 1.6-34.0, $P=0.013$ ) and to find interval monitoring (CA125/USS) between the two surgeries acceptable $(\mathrm{OR}=8.9,95 \% \mathrm{CI}$ $1.5-71.8, P=0.025)$. Lower acceptability of precision of OC risk reduction following RRES $(\mathrm{OR}=0.2,95 \%$ CI $0.1-0.4$, $P<0.0005)$ was the predominant factor affecting premenopausal women who were 'not sure' about undergoing RRESDO compared with those responding 'yes'. When asked about the acceptability of complication rates, 157 (80.1\%) premenopausal women found the complication rate with two procedures acceptable; 125 (87.4\%) women who would undergo RRESDO found this acceptable versus 32 (60.4\%) women who would not undergo RRESDO.

When women who had undergone premenopausal RRSO were asked whether they would have considered undergoing RRESDO instead of RRSO had it been offered (retrospective acceptability), 38.4\% (61/159) and 61.6\% (98/159) responded 'probably/maybe' and 'probably not/definitely not', respectively (Table 2). Multiple logistic-regression model outputs showing association of covariates with retrospective RRESDO acceptability following premenopausal RRSO are given in Table 4. Genetic-mutation type or carrier-status/FH/prior knowledge of tubal origin of OC/ethnicity/education/income did not statistically significantly affect retrospective acceptability of undergoing RRESDO. Following premenopausal RRSO, women who experienced night sweats $(\mathrm{OR}=13.8,95 \%$ CI $1.7-140.2, P=0.018)$, sleep disturbance $\quad(\mathrm{OR}=18.8, \quad 95 \% \quad \mathrm{CI} \quad 3.2-160.1$, $P=0.003)$, sexual dysfunction $(\mathrm{OR}=5.3,95 \%$ CI $1.2-$ $27.5, P=0.031)$ or urinary incontinence $(\mathrm{OR}=17.2,95 \%$ CI 4-98.6, $P<0.001)$ regretted their decision to undergo RRSO (OR $=6.4,95 \%$ CI $1.3-40.7, P=0.032)$; they also felt the decision to undergo RRSO did them a lot of harm $(\mathrm{OR}=3.9,95 \%$ CI $1.2-12.8, P=0.022)$ and were statistically significantly more likely to have chosen retrospectively to undergo RRESDO instead of RRSO had it been available. However, women who experienced hot flushes $(\mathrm{OR}=$ $0.1,95 \%$ CI $0-0.6, P=0.013$ ), osteoporosis ( $\mathrm{OR}=0.3$, $95 \%$ CI $0.1-0.9, P=0.045)$ or fatigue (OR $=0.01,95 \% \mathrm{CI}$ $0-0.1, \quad P<0.001)$ following premenopausal RRSO were
Table 2. RRESDO acceptability

\begin{tabular}{|c|c|}
\hline Acceptability & $n(\%)$ \\
\hline \multicolumn{2}{|c|}{$\begin{array}{l}\text { Acceptability of undergoing RRESDO among premenopausal } \\
\text { women who have not undergone RRSO }\end{array}$} \\
\hline Yes & $145 / 262(55.34)$ \\
\hline No & $53 / 262(20.23)$ \\
\hline Not sure & $64 / 262(24.43)$ \\
\hline \multirow{2}{*}{\multicolumn{2}{|c|}{$\begin{array}{l}\text { Acceptability of participating in a research study offering } \\
\text { RRESDO among premenopausal women who have not } \\
\text { undergone RRSO }\end{array}$}} \\
\hline & \\
\hline Probably & 95/262 (36.26) \\
\hline Maybe & $86 / 262(32.82)$ \\
\hline Probably not & 48/262 (18.32) \\
\hline Definitely not & $33 / 262(12.60)$ \\
\hline \multicolumn{2}{|c|}{$\begin{array}{l}\text { Retrospective acceptability of RRESDO among women who } \\
\text { have undergone premenopausal RRSO }\end{array}$} \\
\hline Probably & 28/159 (17.61) \\
\hline Maybe & $33 / 159(20.75)$ \\
\hline Probably not & $46 / 159$ (28.93) \\
\hline Definitely not & $52 / 159(32.70)$ \\
\hline
\end{tabular}

RRESDO, risk-reducing early salpingectomy with delayed oophorectomy; RRSO, risk-reducing salpingo-oophorectomy.

statistically significantly less likely to have chosen to have undergone RRESDO instead of RRSO retrospectively.

Overall, HRT use among premenopausal women postRRSO was $53.7 \%$ (88/164). However, when stratified by BC status at the time of premenopausal RRSO, the HRT uptake was $74.1 \%(80 / 108)$ among BC-unaffected and $14.3 \%(8 / 56)$ among BC-affected individuals. The mean age at premenopausal RRSO was $47.2(\mathrm{SD}=6.7)$ years and the mean duration of HRT use was $4.2(\mathrm{SD}=3)$ years. No woman who had undergone postmenopausal RRSO used HRT. The multiple logistic-regression model explored the association of covariates with HRT use versus non-use following premenopausal RRSO. Women with a university level (versus below university) education ( $\mathrm{OR}=3.1,95 \%$ CI $1.2-8.5, P=0.021)$ and women who were aware/concerned about neurocognitive decline $(\mathrm{OR}=11.2,95 \% \mathrm{CI}$ $1.2-136.8, P=0.045)$ were significantly more likely to use HRT. There was no difference in HRT use versus non-use in women who had undergone premenopausal RRSO who were experiencing sexual dysfunction.

Table S2 reports satisfaction/regret with the RRSO decision making by menopausal status at the time of surgery. There was high satisfaction ('agree/strongly agree' responses) among women who had undergone premenopausal and postmenopausal RRSO (88.82\% [143/161] and 95.24\% [80/ 84 ], respectively, $P=0.635)$ and a similarly high proportion would make the same decision again $(87.6 \%$ [141/161] versus $94 \%$ [79/84], $P=0.186)$. When compared with women undergoing postmenopausal RRSO, those undergoing 
Gaba et al.

premenopausal RRSO had a statistically significantly higher regret rate (respectively 9.4\% [15/160] versus $1.2 \%$ [1/81], $P=0.008$ ) and were more likely to feel that RRSO did them a lot of harm $(11.25 \%$ [18/160] versus 5\% [4/80], $P=0.006)$. Multiple linear-regression modelling showed that HRT use did not statistically significantly affect satisfaction/regret levels in premenopausal women. Correlation analysis did not find a statistically significant association between satisfaction/regret and age of premenopausal RRSO $(r=-0.085, P=0.292)$. Multiple linear-regression model outputs showing an association of menopausal sequelae with satisfaction/regret following premenopausal RRSO are given in Table S3. Women experiencing menopausal sequelae (sexual dysfunction, night sweats, sleep disturbance, vaginal dryness, urinary incontinence, fatigue, memory loss, mood alterations, negative impact on relationship with partner) were significantly more likely to regret undergoing premenopausal RRSO. The multiple logistic-regression model explored the association of HRT use with non-use on menopausal sequelae after premenopausal RRSO (Table S4). HRT users (compared with non-users) were significantly less likely to experience vaginal dryness $(\mathrm{OR}=0.4,95 \%$ CI $0.2-0.9$, $P=0.025)$ but the prevalence of other symptoms was not reduced.

Table 3. Factors affecting acceptability of undergoing RRESDO among premenopausal women who have not undergone RRSO

\begin{tabular}{|c|c|c|c|c|}
\hline & OR & SE & $P>|z|$ & $95 \% \mathrm{Cl}$ \\
\hline Prior knowledge of tubal origin of $O C$ & 1.725 & 0.376 & 0.147 & $0.833-3.655$ \\
\hline Personal history of $\mathrm{BC}$ & 1.184 & 0.558 & 0.761 & $0.404-3.672$ \\
\hline Previous RRM & 0.647 & 0.407 & 0.285 & $0.286-1.421$ \\
\hline Family complete & 1.146 & 0.516 & 0.792 & $0.417-3.214$ \\
\hline Carrier status & 1.788 & 0.911 & 0.523 & $0.273-10.928$ \\
\hline Marital status & 0.679 & 0.425 & 0.362 & $0.289-1.545$ \\
\hline Ethnicity & 0.492 & 0.599 & 0.237 & $0.153-1.66$ \\
\hline Education & 0.876 & 0.408 & 0.747 & $0.388-1.94$ \\
\hline Income & 1.053 & 0.177 & 0.77 & $0.742-1.492$ \\
\hline Timing of future $\mathrm{OC}$ prevention surgery & 0.286 & 1.171 & 0.286 & $0.014-2.095$ \\
\hline \multicolumn{5}{|l|}{ Family history } \\
\hline $\mathrm{BC}$ & 2.889 & 0.63 & 0.093 & $0.813-9.999$ \\
\hline $\mathrm{OC}$ & 1.019 & 0.785 & 0.98 & $0.214-4.799$ \\
\hline$B C$ and $O C$ & 0.869 & 0.867 & 0.871 & $0.158-4.835$ \\
\hline \multicolumn{5}{|c|}{ Concerns over premature menopause sequelae influencing decision to undergo RRESDO } \\
\hline Hot flushes/night sweats & 1.27 & 0.502 & 0.634 & $0.475-3.451$ \\
\hline Looking older & 0.804 & 0.5 & 0.663 & $0.295-2.131$ \\
\hline Decreased libido/other sexual side effects & 2.918 & 0.477 & 0.025 & $1.163-7.648$ \\
\hline Loss of fertility & 1.568 & 0.608 & 0.459 & $0.468-5.208$ \\
\hline Osteoporosis & 1.931 & 0.567 & 0.246 & $0.628-5.895$ \\
\hline Heart disease & 0.845 & 0.625 & 0.787 & $0.239-2.832$ \\
\hline Dementia/memory dysfunction & 2.435 & 0.67 & 0.184 & $0.67-9.495$ \\
\hline Impact on survival & 0.488 & 0.511 & 0.16 & $0.17-1.281$ \\
\hline Acceptability of having to take HRT until 51 years & 1.501 & 0.437 & 0.353 & $0.64-3.586$ \\
\hline \multicolumn{5}{|c|}{ Potential benefits of RRESDO influencing decision to undergo RRESDO } \\
\hline Reduces risk of $\mathrm{OC}$ without premature menopause & 9.007 & 1.195 & 0.066 & $1.149-192.856$ \\
\hline Inspection of tubes/ovaries by doctor & 2.323 & 0.798 & 0.291 & $0.474-11.476$ \\
\hline delays hot flushes, night sweats & 5.028 & 0.719 & 0.025 & $1.218-21.172$ \\
\hline delays osteoporosis & 1.08 & 1.332 & 0.954 & $0.083-17.385$ \\
\hline delays potential change to sexual function & 2.945 & 0.735 & 0.142 & $0.682-12.753$ \\
\hline Not associated with increased risk of heart disease & 1.279 & 1.127 & 0.827 & $0.114-11.238$ \\
\hline \multicolumn{5}{|c|}{ Potential limitations of RRESDO influencing decision to undergo RRESDO } \\
\hline Two staged surgery & 444.078 & 1.672 & $<0.001$ & *28.04- 22814.9 \\
\hline Potential premature menopause & 1.939 & 0.888 & 0.456 & $0.348-12.145$ \\
\hline Increased complication rate & 0.78 & 1.014 & 0.807 & $0.091-5.333$ \\
\hline Interval monitoring between surgeries & 59.027 & 1.471 & 0.006 & $4.221-1548.671$ * \\
\hline Additional time in hospital & 0.028 & 1.94 & 0.065 & $0-1.081$ \\
\hline Additional time off work for surgery/post-operative recovery & 6.166 & 1.453 & 0.21 & $0.406-139.284$ \\
\hline
\end{tabular}


Table 3. (Continued)

\begin{tabular}{lcccc}
\hline & OR & SE & P > |z| & 95\% CI \\
\hline Precise level of OC risk reduction with ES unknown & 14.556 & 1.095 & 0.015 & $\begin{array}{l}1.961-160.637 \\
1.405-93.72\end{array}$ \\
Developing an interval OC between the two surgeries & 9.554 & 1.05 & 0.032 & 1.032 \\
\hline
\end{tabular}

BC, breast cancer; FH, family history; HRT, hormone replacement therapy; OC, ovarian cancer; RRESDO, risk-reducing early salpingectomy with delayed oophorectomy; RRM, risk-reducing mastectomy.

Multiple logistic regression analysis on factors affecting acceptability of undergoing RRESDO ('yes' versus 'no' responses) in 198 premenopausal women who have not undergone RRSO. Model adjusted for marital status, ethnicity, education, income, family history of ovarian cancer/breast cancer, risk-reducing mastectomy and personal history of breast cancer.

Prior knowledge of tubal origin of OC: 'yes' versus 'no'; personal history of BC: 'yes' versus 'no'; previous RRM: 'yes' versus 'no'; family complete: 'no' versus 'yes'; carrier status: BRCA1/BRCA2 versus intermediate risk (RAD51C carrier/RAD51D carrier/BRIP1 carrier/BRCA negative but strong FH of OC/BRCA untested but strong FH of OC); marital status: in a relationship (married, cohabiting/living with partner) versus not in a relationship (single, divorced, separated, widowed); ethnicity: non-caucasian versus caucasian; education: university level education (PhD, Masters, Bachelor's degree) versus below university level education (NVQ4, A-level/NVQ3, NVQ1/NVQ2, GCSE/O-level/CSE, no formal qualification); timing of future $O C$ prevention surgery: planning surgery now/within 5 years versus not planning surgery; $F H B C$ (FH of BC alone plus FH of BC and OC): 'yes' versus ' $\mathrm{no}$; ; $\mathrm{FH} \mathrm{OC} \mathrm{(FH} \mathrm{of} \mathrm{OC} \mathrm{alone} \mathrm{plus} \mathrm{FH}$ of OC and BC): 'yes' versus 'no'; FH BC and OC: 'yes' versus 'no'; hot flushes/night sweats: 'yes' versus 'no'; looking older: 'yes' versus 'no'; decreased libido/other sexual side effects: 'yes' versus 'no'; loss of fertility: 'yes' versus 'no'; osteoporosis (self-reported): 'yes' versus 'no'; heart disease: 'yes' versus 'no'; dementia/memory dysfunction: 'yes' versus 'no'; impact on survival: 'yes' versus 'no'; acceptability of having to take HRT until 51 years: 'yes' versus 'no'; reduces risk of OC without premature menopause: 'yes' versus 'no'; inspection of tubes/ovaries by doctor: 'yes' versus 'no'; delays hot flushes/night sweats: 'yes' versus 'no'; delays osteoporosis: 'yes' versus 'no'; delays potential change to sexual function: 'yes' versus 'no'; not associated with increased risk of heart disease: 'yes' versus 'no'; two-stage surgery: 'yes' versus 'no'; potential premature menopause: 'yes' versus 'no'; increased complication rate: 'yes' versus 'no'; interval monitoring between surgeries: 'yes' versus 'no'; additional time in hospital: 'yes' versus 'no'; additional time off work for surgery/postoperative recovery: 'yes' versus 'no'; precise level of OC risk reduction with ES unknown: 'yes' versus 'no'; developing an interval OC between the two surgeries: 'yes' versus 'no'.

*Extreme value of some upper limits of confidence intervals indicate that there were too few responses in some categories of responses.

Table S5 reports the prevalence of menopausal sequelae following pre- and postmenopausal RRSO. The prevalence of sequelae in premenopausal women ranged from 50 to $74.1 \%$, with $66.3 \%$ of women experiencing sexual dysfunction and 66.9-74.1\% experiencing hot flushes/night sweats/ sleep disturbance. Symptoms were significantly more frequent following premenopausal than following menopausal RRSO (Table S5).

\section{Discussion}

\section{Main findings}

Putative acceptability of undergoing RRESDO among premenopausal women who have not undergone OC prevention surgery is $55.3 \%$, and $69.1 \%$ would find participating in a research study offering RRESDO acceptable. Premenopausal women who have not undergone OC-prevention surgery and were concerned about/wanted to delay sexual dysfunction were statistically significantly more likely to find undergoing RRESDO acceptable and participate in a research study offering RRESDO. Similarly, women who had undergone premenopausal RRSO and experienced sexual dysfunction/ vasomotor symptoms/urinary incontinence were significantly more likely to regret their decision and find RRESDO acceptable retrospectively. In all, $38.4 \%$ of women who underwent premenopausal RRSO would retrospectively have found RRESDO acceptable were it an option. Although satisfaction with undergoing RRSO was high (premenopausal $\mathrm{RRSO}=88.82 \%$, postmenopausal $\quad \mathrm{RRSO}=95.24 \%$ ) for women undergoing premenopausal RRSO, 9.38\% regretted their decision and $11.25 \%$ felt it did them a lot of harm. Our data suggest good HRT compliance, with $74 \%$ of BC-unaffected women using HRT until the recommended age of 51. HRT use did not statistically significantly affect satisfaction/ regret levels or alleviate menopausal symptoms except vaginal dryness among women undergoing premenopausal RRSO. There was a higher prevalence of menopausal sequelae following premenopausal RRSO. Women who experienced menopausal sequelae following premenopausal RRSO had higher regret levels.

\section{Strengths and weaknesses}

Strengths include that participants were informed in detail about OC-prevention surgery with a detailed participant information-sheet prior to completing our questionnaire; ours is the only study to have generated UK data on RRESDO acceptability and menopausal sequelae/satisfaction/regret following RRSO and it is the largest study internationally reporting RRESDO acceptability. Limitations include that the proportions of individuals with a 
Gaba et al.

Table 4. Factors associated with retrospective acceptability of RRESDO among women who have undergone premenopausal RRSO

\begin{tabular}{|c|c|c|c|c|}
\hline & OR & SE & $P>|z|$ & $95 \% \mathrm{Cl}$ \\
\hline Prior knowledge of tubal origin of ovarian cancer & 1.514 & 0.444 & 0.35 & $0.63-3.625$ \\
\hline Personal history of breast cancer & 0.731 & 0.468 & 0.504 & $0.289-1.824$ \\
\hline Previous risk reducing mastectomy & 0.618 & 0.456 & 0.291 & $0.249-1.5$ \\
\hline Carrier status & 1.626 & 0.898 & 0.589 & $0.31-12.385$ \\
\hline Marital status & 0.716 & 0.458 & 0.466 & $0.291-1.768$ \\
\hline Ethnicity & 1.184 & 0.635 & 0.791 & $0.321-4.058$ \\
\hline Education & 1.11 & 0.398 & 0.793 & $0.507-2.433$ \\
\hline Income & 1.079 & 0.194 & 0.695 & $0.739-1.589$ \\
\hline \multicolumn{5}{|l|}{ FH } \\
\hline BC & 1.445 & 0.896 & 0.682 & $0.281-11.041$ \\
\hline OC & 1.754 & 1.120 & 0.616 & $0.198-18.561$ \\
\hline$B C$ and $O C$ & 0.944 & 1.166 & 0.961 & $0.083-9.194$ \\
\hline \multicolumn{5}{|l|}{ Experienced menopausal sequelae following RRSO } \\
\hline Hot flushes & 0.09 & 0.955 & 0.013 & $0.013-0.566$ \\
\hline Night sweats & 13.76 & 1.108 & 0.018 & $1.729-140.177$ \\
\hline Sleep disturbance & 18.78 & 0.988 & 0.003 & $3.186-160.144$ \\
\hline Vaginal dryness & 0.76 & 0.658 & 0.671 & $0.204-2.774$ \\
\hline Sexual dysfunction & 5.34 & 0.779 & 0.031 & $1.244-27.467$ \\
\hline Loss of fertility & 0.70 & 0.669 & 0.587 & $0.174-2.501$ \\
\hline Osteoporosis & 0.25 & 0.700 & 0.045 & $0.057-0.919$ \\
\hline Urinary incontinence & 17.20 & 0.807 & $<0.001$ & 3.985-98.597 \\
\hline Fatigue & 0.01 & 1.150 & $<0.001$ & $0.001-0.096$ \\
\hline Memory loss & 0.67 & 0.662 & 0.541 & $0.177-2.45$ \\
\hline Mood alterations & 3.06 & 0.684 & 0.102 & $0.834-12.654$ \\
\hline Impact on relationship with partner & 1.81 & 0.676 & 0.379 & $0.49-7.158$ \\
\hline \multicolumn{5}{|l|}{ Satisfaction/regret following RRSO } \\
\hline I regret the choice that was made & 6.385 & 0.866 & 0.032 & $1.251-40.746$ \\
\hline I would make the same decision if I had to do it over again & 0.471 & 4.597 & 0.87 & $0.001-205.952$ \\
\hline The decision did me a lot of harm & 3.877 & 0.590 & 0.022 & $1.226-12.795$ \\
\hline I was directly influenced by the direct wishes of my family & 3.762 & 0.700 & 0.058 & $0.974-15.615$ \\
\hline I was influenced by the unexpressed wishes of my family & 0.401 & 0.734 & 0.212 & $0.089-1.631$ \\
\hline
\end{tabular}

BC, breast cancer; $F H$, family history; HRT, hormone replacement therapy; OC, ovarian cancer; RRESDO, risk-reducing early salpingectomy with delayed oophorectomy; RRM, risk-reducing mastectomy.

Multiple logistic regression analysis on factors affecting retrospective acceptability ('probably, maybe' versus 'probably not, definitely not' responses) of undergoing RRESDO had it been an option in 159 women who have undergone premenopausal RRSO. Model adjusted for marital status, ethnicity, education, income, family history of ovarian cancer/breast cancer, risk-reducing mastectomy and personal history of breast cancer. Prior knowledge of tubal origin of OC: 'yes' versus 'no'; personal history of BC: 'yes' versus 'no'; previous RRM: 'yes' versus 'no'; family complete: no versus yes; carrier status: BRCA1/BRCA2 versus intermediate risk (RAD51C carrier/RAD51D carrier/BRIP1 carrier/BRCA-negative but strong FH of OC/BRCA untested but strong FH of OC); marital status: in a relationship (married, cohabiting/living with partner) versus not in a relationship (single, divorced, separated, widowed); ethnicity: non-caucasian versus caucasian; education: university level education (PhD, Masters, Bachelor's degree) versus below university level education (NVQ4, A-level/NVQ3, NVQ1/NVQ2, GCSE/O-level/CSE, no formal qualification); timing of future $\mathrm{OC}$ prevention surgery: planning surgery now/within 5 years versus not planning surgery; $F H B C$ ( $F H$ of $B C$ alone plus $F H$ of $B C$ and $\mathrm{OC}$ ): 'yes' versus 'no'; FH OC (FH of OC alone plus FH of OC and BC): 'yes' versus 'no'; FH BC and OC: 'yes' versus 'no'; hot flushes: 'yes' versus 'no'; night sweats: 'yes' versus 'no'; sleep disturbance: 'yes' versus 'no'; vaginal dryness: 'yes' versus 'no'; sexual dysfunction: 'yes' versus 'no'; loss of fertility: 'yes' versus 'no'; osteoporosis (self-reported): 'yes' versus 'no'; urinary incontinence: 'yes' versus 'no'; fatigue: 'yes' versus 'no'; memory loss: 'yes' versus 'no'; mood alterations: 'yes' versus 'no'; impact of relationship with partner: 'yes' versus 'no'; it was the right decision: 'yes' versus 'no'; I regret the choice that was made: 'yes' versus 'no'; I would make the same decision if I had to do it over again: 'yes' versus 'no'; the decision did me a lot of harm: 'yes' versus 'no'; the decision was a wise one: 'yes' versus 'no'; I was directly influenced by the direct wishes of my family: 'yes' versus 'no'; I was influenced by the unexpressed wishes of my family: 'yes' versus 'no'.

university degree/household income of $\geq$ UK£ 30,000 were higher than the UK national average and $88 \%$ of our cohort were caucasian. Consequently, these results may not be generalisable to a minority, lesser educated/affluent population of BRCA-carriers. Also, because only $1.77 \%$ $(12 / 683)$ of our cohort were at intermediate OC risk, no inferences can be drawn for this subgroup. In addition, questionnaire responses may have been affected by recall 
bias. We excluded women with a previous history of OC so as not to bias results, as they do not reflect unaffected women considering prophylactic OC-surgery.

\section{Interpretation}

A US survey-study reported 34.3\% (70/204) acceptability in taking part in a research study offering RRESDO among premenopausal women who had not undergone RRSO. ${ }^{36}$ This is lower than the $69.1 \%(181 / 262)$ we found, despite both cohorts being similar in terms of baseline demographics (mean age 38.25 versus 35.4 years; 88 versus $90.7 \%$ caucasian, personal history of BC 22.8 versus $16.7 \%$ ) and our cohort having more nulliparous women (64.6 versus $41.2 \%$ ). Differences in healthcare systems (UK state-funded versus US-privatised/insurance-based) and increasing awareness may have contributed to the dissimilar findings. In the USA, $32.8 \%(20 / 61)$ of women not interested in participating in a research study offering RRESDO, cited concerns related to additional healthcare costs as their reason to decline participation. ${ }^{36}$

The lack of a precise level of OC risk-reduction data with ES was an important limitation for premenopausal women who had not undergone OC-prophylactic surgery and who did not find RRESDO acceptable. Additionally, impaired sexual function, as a direct consequence of premenopausal-oophorectomy, is an extremely important consideration for women who have not undergone surgical prevention. There are currently three trials open to recruitment, investigating aspects of RRESDO. ${ }^{40}$ The UK trial PROTECTOR $^{33}$ and US trial WISP ${ }^{35}$ are both powered on sexual function and will provide valuable information on the impact of RRESDO on sexual function. That women experiencing night sweats/urinary incontinence/sexual dysfunction were significantly more likely to find RRESDO acceptable but those experiencing other symptoms might not, suggests there is a range of tolerability and acceptability of various symptoms among women which affects surgical decision making.

Our data show $50.7 \%(346 / 683)$ RRSO uptake, which is consistent with literature reports of $12-78 \% .^{14,24,27,36,41-68}$ Uptake was higher among older women, women who completed childbearing, those who had BC themselves, and those having undergone RRM. This is in keeping with published data. ${ }^{41,44,47,50,57,62,69,70}$

Offering RRESDO to premenopausal women who have completed childbearing may reduce uptake of premenopausal RRSO. This is supported by our data, which show that $38.4 \%$ of women who had undergone premenopausal RRSO would have in retrospect found RRESDO acceptable had it been an option. However, RRESDO may increase the overall number of women undergoing premenopausal OC surgical prevention, as it offers an alternative option to individuals otherwise declining premenopausal oophorectomy due to the negative consequences of premature menopause.

Satisfaction following RRSO in BRCA-carriers is reported to be high at $86-97 \%$ at $1-3$ years post-surgery, ${ }^{27,46,66,71,72}$ which is in keeping with our results. However, 9.4\% (15/ 150) women in our cohort who underwent premenopausal RRSO regretted their decision, which is higher than the 5$7 \%$ reported 2-3 years post-RRSO in the literature. ${ }^{27,71}$ Our data show a positive correlation between regret and menopausal sequelae following surgery. However, these results must be interpreted with caution in light of the small number of women $(n=15)$ who reported regret. There are no published data on the effect of HRT on satisfaction/regret following RRSO. Our results show that HRT use did not statistically significantly affect satisfaction/regret levels in women who had undergone premenopausal RRSO despite a high HRT uptake of $74.1 \%$ in BC-unaffected women who have undergone premenopausal RRSO. HRT uptake in women undergoing premenopausal RRSO is reported to be $8-75 \%$ in the literature. ${ }^{28,72-75}$ Although our reported uptake is at the upper end of this range, it may not be a true reflection of practice in the UK. We acquired our data through specialist familial cancer clinics which manage women in a multidisciplinary setting that includes routine counselling on the detrimental health sequelae of premature menopause along with the importance of HRT in mitigating these risks and input/access to menopause specialists for symptom and sequelae management. A large proportion of UK women at increased OC risk are managed in non-specialist settings and may receive inconsistent menopause management advice. ${ }^{76}$ Short-term HRT use following premenopausal RRSO in unaffected BRCA1/BRCA2carriers is beneficial. ${ }^{77-80}$ Premenopausal women should be involved in formulating an individualised pre-surgical menopause symptom management and HRT-plan and fully counselled regarding the consequences of iatrogenic menopause, treatment benefits and its efficacy in alleviating vasomotor symptoms and sexual dysfunction. This needs to be discussed as part of informed consent and incorporated into the RRSO decision-making process.

Contrary to our data, the literature shows that HRT use following premenopausal RRSO reduces the prevalence and severity of hot flushes. ${ }^{28,75,81,82}$ However, consistent with our data, according to the literature, HRT use following premenopausal RRSO reduces vaginal dryness but does not alleviate sexual dysfunction (sexual pleasure/habit/satisfaction/libido). ${ }^{28,72,75,81}$

The literature reports that women undergoing premenopausal RRSO experience a significant worsening of endocrine symptoms (hot flushes/night sweats/sweating). ${ }^{72}$ However, the literature on endocrine symptoms following postmenopausal RRSO is conflicting with different studies reporting either no increase ${ }^{72}$ or increase in the prevalence 
of endocrine symptoms. ${ }^{27}$ In our study, baseline levels of menopausal sequelae symptoms prior to premenopausal and postmenopausal RRSO were not recorded, and although our data show a prevalence of $66.9-74.1 \%$ and $25.4-40.7 \%$ of endocrine symptoms following pre- and postmenopausal RRSO, respectively, we cannot be certain some symptoms were not already present prior to RRSO or whether they worsened following surgery, particularly in postmenopausal women.

\section{Conclusion}

Acceptance of the central role of the fallopian tube in aetiopathogenesis of OC and health consequences of premature menopause from oophorectomy has led to RRESDO being proposed as a surgical alternative for premenopausal women whose family is complete but who decline/delay oophorectomy. Given lack of data on long-term health, the extent of OC risk reduction and concerns over attrition, RRESDO is currently recommended only within the context of a research trial. A total of $69.1 \%$ of UK women who have not undergone premenopausal oophorectomy and particularly women concerned about sexual dysfunction would find it acceptable to participate in a research study offering RRESDO. Although RRSO remains the gold standard for OC prevention, $\sim 10 \%$ who undergo premenopausal RRSO regret their decision, particularly due to menopausal sequelae. HRT uptake is good in women managed in specialist centres. Although HRT use has been shown to mitigate some endocrine symptoms, it does not appear to alleviate sexual dysfunction or increase satisfaction levels following RRSO. Women at increased OC risk contemplating OC-prevention surgery may be better managed in a multidisciplinary setting of specialist familial cancer clinics with input from gynaecologists/psychologists/menopause/fertility specialists with links to genetics teams.

\section{Disclosure of interests}

RM declares research funding from Barts \& the London Charity and Eve Appeal outside this work, an honorarium for grant review from Israel National Institute for Health Policy Research and honorarium for advisory board membership from Astrazeneca/MSD. RM is supported by an NHS Innovation Accelerator (NIA) Fellowship for population testing. UM has a financial interest in Abcodia, Ltd., a company formed to develop academic and commercial development of biomarkers for screening and risk prediction. DGE declares honoraria for advisory board membership from Astrazeneca. The other authors declare no conflict of interest. Completed disclosure of interest forms are available to view online as supporting information.

\section{Contribution to authorship}

Conception: RM. Design \& development: FG, RM. Questionnaire development: FG, DC, CG, UM, RL, RM. Data collection: FG, DC, MO, SG, RM, LI, VT, IE, LM, KR, RC, GE. Data analysis: FG, OB, RM. Preparation of tables: FG, OB. Initial draft of manuscript: FG, RM. Manuscript writing, review and approval: All authors.

\section{Details of ethics approval}

The RRESDO Survey study received full ethics approval from West Midlands - Edgbaston Research Ethics Committee on 21 August 2017 (REC Reference number 17/WM/ 0324). The study was registered with the International Standard Randomized Controlled Trial Number Register ISRCTN $12310993 \quad$ (https://doi.org/10.1186/ISRCTN1 2310993). All study volunteers provided written informed consent to participate in the study.

\section{Funding}

This work underwent peer-review and was supported by Rosetrees Trust (grant number M779). The funding body had no role in the study design, data collection, analysis, interpretation or writing of the report or decision to submit for publication. The UK PROTECTOR study into early salpingectomy in high-risk women is supported by The Barts Charity (grant MRC0167). The research team was independent of funders.

The funding body had no role in the study design, data collection, analysis, interpretation or writing of the report or decision to submit for publication. The research team was independent of funders.

\section{Acknowledgements}

The study is supported by researchers at the Barts Cancer Research UK Centre for Excellence, Queen Mary University of London (C16420/A18066). We are particularly grateful to the women who participated in the study. We are grateful to the entire medical, nursing and administrative staff who work on the RRESDO Survey Study. We are grateful to BRCA Umbrella for increasing awareness of our study. We are grateful to Barts Health NHS Trust, University College London Hospitals NHS Foundation Trust, Guy's and St Thomas' NHS Foundation Trust, Manchester University NHS Foundation Trust, Cambridge University Hospitals NHS Foundation Trust and NHS Tayside for their support of the study. We are grateful to Nicola Flaum and Robert D. Morgan for their support. DGRE is supported by the Manchester National Institute for Health Research Biomedical Research Centre (IS-BRC-121520007). NF and RDM are supported by Cancer Research UK. 


\section{Data sharing}

Relevant anonymised data can be obtained on reasonable request from the corresponding author.

\section{Supporting Information}

Additional supporting information may be found online in the Supporting Information section at the end of the article.

Table S1. Factors affecting 'not sure' versus 'no' questionnaire responses on acceptability of undergoing RRESDO among premenopausal women who have not undergone RRSO.

Table S2. Satisfaction and regret according to menopausal status at the time of RRSO.

Table S3. Effect of menopausal sequelae on satisfaction and regret among women who have undergone premenopausal RRSO.

Table S4. Effect off HRT use versus non-use on menopausal sequelae in women who have undergone premenopausal RRSO.

Table S5. Prevalence of menopausal sequelae by menopausal status at the time off RRSO.

Appendix S1. Participant information sheet: Views of women at high risk of ovarian cancer towards removal of fallopian tubes for ovarian cancer prevention.

Appendix S2. Survey to determine attitudes of women at increased risk of ovarian cancer towards risk-reducing early salpingectomy with delayed oophorectomy for ovarian cancer prevention.

\section{References}

1 Barrow E, Hill J, Evans DG. Cancer risk in Lynch Syndrome. Fam Cancer 2013;12:229-40

2 Antoniou A, Pharoah PD, Narod S, Risch HA, Eyfjord JE, Hopper JL, et al. Average risks of breast and ovarian cancer associated with BRCA1 or BRCA2 mutations detected in case Series unselected for family history: a combined analysis of 22 studies. Am J Hum Genet 2003; 72:1117-30.

3 Chen S, Parmigiani G. Meta-analysis of BRCA1 and BRCA2 penetrance. J Clin Oncol 2007;25:1329-33.

4 Kuchenbaecker KB, Hopper JL, Barnes DR, Phillips KA, Mooij TM, Roos-Blom MJ, et al. Risks of breast, ovarian, and contralateral breast cancer for BRCA1 and BRCA2 mutation carriers. JAMA 2017;317:2402-16.

5 Evans DG, Shenton A, Woodward E, Lalloo F, Howell A, Maher ER. Penetrance estimates for BRCA1 and BRCA2 based on genetic testing in a Clinical Cancer Genetics service setting: risks of breast/ ovarian cancer quoted should reflect the cancer burden in the family. BMC Cancer 2008;8:155.

6 Loveday C, Turnbull C, Ramsay E, Hughes D, Ruark E, Frankum JR, et al. Germline mutations in RAD51D confer susceptibility to ovarian cancer. Nat Genet 2011;43:879-82.

7 Loveday C, Turnbull C, Ruark E, Xicola RM, Ramsay E, Hughes D, et al. Germline RAD51C mutations confer susceptibility to ovarian cancer. Nat Genet 2012;44:475-6.
8 Ramus SJ, Song H, Dicks E, Tyrer JP, Rosenthal AN, Intermaggio MP, et al. Germline mutations in the BRIP1, BARD1, PALB2, and NBN genes in women with ovarian cancer. J Nat/ Cancer Inst 2015;107:djv214.

9 Yang X, Leslie G, Doroszuk A, Schneider S, Allen J, Decker B, et al. Cancer risks associated with germline PALB2 pathogenic variants: an international study of 524 families. J Clin Oncol 2019;38:674-685.

10 Yang $X$, Song $H$, Leslie G, Engel C, Hahnen E, Auber B, et al. Ovarian and breast cancer risks associated with pathogenic variants in RAD51C and RAD51D. J Natl Cancer Inst 2020;112:1-9.

11 Manchanda R, Legood R, Antoniou AC, Pearce L, Menon U. Commentary on changing the risk threshold for surgical prevention of ovarian cancer. BJOG 2017;125:541-4.

12 Manchanda R, Legood R, Antoniou AC, Gordeev VS, Menon U. Specifying the ovarian cancer risk threshold of 'premenopausal riskreducing salpingo-oophorectomy' for ovarian cancer prevention: a cost-effectiveness analysis. J Med Genet 2016;53:591-9.

13 Manchanda R, Legood R, Pearce L, Menon U. Defining the risk threshold for risk reducing salpingo-oophorectomy for ovarian cancer prevention in low risk postmenopausal women. Gynecol Oncol 2015;139:487-94.

14 Kauff ND, Satagopan JM, Robson ME, Scheuer L, Hensley M, Hudis CA, et al. Risk-reducing salpingo-oophorectomy in women with a BRCA1 or BRCA2 mutation. N Engl J Med 2002;346:1609-15.

15 Rebbeck TR, Lynch HT, Neuhausen SL, Narod SA, Van't Veer L, Garber JE, et al. Prophylactic oophorectomy in carriers of BRCA1 or BRCA2 mutations. N Engl J Med 2002;346:1616-22.

16 Kauff ND, Domchek SM, Friebel TM, Robson ME, Lee J, Garber JE, et al. Risk-reducing salpingo-oophorectomy for the prevention of BRCA1- and BRCA2-associated breast and gynecologic cancer: a multicenter, prospective study. J Clin Oncol 2008;26:1331-7.

17 Finch A, Beiner M, Lubinski J, Lynch HT, Moller P, Rosen B, et al. Salpingo-oophorectomy and the risk of ovarian, fallopian tube, and peritoneal cancers in women with a BRCA1 or BRCA2 mutation. JAMA 2006;296:185-92.

18 Rebbeck TR, Kauff ND, Domchek SM. Meta-analysis of risk reduction estimates associated with risk-reducing salpingooophorectomy in BRCA1 or BRCA2 mutation carriers. J Natl Cancer Inst 2009;101:80-7.

19 Eisen A, Lubinski J, Klijn J, Moller P, Lynch HT, Offit K, et al. Breast cancer risk following bilateral oophorectomy in BRCA1 and BRCA2 mutation carriers: an international case-control study. J Clin Oncol 2005;23:7491-6.

20 Domchek SM, Friebel TM, Singer CF, Evans DG, Lynch HT, Isaacs C, et al. Association of risk-reducing surgery in BRCA1 or BRCA2 mutation carriers with cancer risk and mortality. JAMA 2010;304:967-75.

21 Heemskerk-Gerritsen BA, Seynaeve C, van Asperen CJ, Ausems MG, Collee JM, van Doorn HC, et al. Breast cancer risk after salpingooophorectomy in healthy BRCA1/2 mutation carriers: revisiting the evidence for risk reduction. J Natl Cancer Inst 2015;107:djv033.

22 Kotsopoulos J, Huzarski T, Gronwald J, Singer CF, Moller P, Lynch $H T$, et al. Bilateral oophorectomy and breast cancer risk in BRCA1 and BRCA2 mutation carriers. J Nat/ Cancer Inst 2016;109:djw177.

23 Meeuwissen PA, Seynaeve C, Brekelmans CT, Meijers-Heijboer HJ, Klijn JG, Burger CW. Outcome of surveillance and prophylactic salpingo-oophorectomy in asymptomatic women at high risk for ovarian cancer. Gynecol Oncol 2005;97:476-82.

24 Garcia C, Lyon L, Conell C, Littell RD, Powell CB. Osteoporosis risk and management in BRCA1 and BRCA2 carriers who undergo riskreducing salpingo-oophorectomy. Gynecol Oncol 2015;138:723-6.

25 Parker WH, Broder MS, Chang E, Feskanich D, Farquhar C, Liu Z, et al. Ovarian conservation at the time of hysterectomy and longterm health outcomes in the nurses' health study. Obstet Gynecol 2009;113:1027-37 
Gaba et al.

26 Rocca WA, Grossardt BR, Shuster LT. Oophorectomy, estrogen and dementia: a 2014 update. Mol Cell Endocrinol 2014;389:712.

27 Madalinska JB, Hollenstein J, Bleiker E, van Beurden M, Valdimarsdottir HB, Massuger LF, et al. Quality-of-life effects of prophylactic salpingo-oophorectomy versus gynecologic screening among women at increased risk of hereditary ovarian cancer. J Clin Oncol 2005;23:6890-8.

28 Madalinska JB, van Beurden M, Bleiker EMA, Valdimarsdottir HB, Hollenstein J, Massuger LF, et al. The impact of hormone replacement therapy on menopausal symptoms in younger high risk women after prophylactic salpingo-oophorectomy. I Clin Oncol 2006:24:3576-82.

29 Schenberg T, Mitchell G. Prophylactic bilateral salpingectomy as a prevention strategy in women at high risk of ovarian cancer: a minireview. Front Oncol 2014;4:21.

30 McAlpine JN, Hanley GE, Woo MM, Tone AA, Rozenberg N, Swenerton KD, et al. Opportunistic salpingectomy: uptake, risks, and complications of a regional initiative for ovarian cancer prevention. Am J Obstet Gynecol 2014;210: 471.e1-e11.

31 Kwon JS, McAlpine JN, Hanley GE, Finlayson SJ, Cohen T, Miller DM, et al. Costs and benefits of opportunistic salpingectomy as an ovarian cancer prevention strategy. Obstet Gynecol 2015;125: 338-45.

32 Chandrasekaran D, Menon U, Evans G, Crawford R, Saridogan E, Jacobs $C$, et al. Risk reducing salpingectomy and delayed oophorectomy in high risk women: views of cancer geneticists, genetic counsellors and gynaecological oncologists in the UK. Fam Cancer 2015; 14:521-30.

33 Barrow E, Robinson L, Alduaij W, Shenton A, Clancy T, Lalloo F, et al. Cumulative lifetime incidence of extracolonic cancers in Lynch syndrome: a report of 121 families with proven mutations. Clin Genet 2009;75:141-9.

34 Bateman BG, Kolp LA, Hoeger K. Complications of laparoscopy - operative and diagnostic. Fertil Steril 1996;66:30-5.

35 Bates T, Kearins O, Monypenny I, Lagord C, Lawrence G. Clinical outcome data for symptomatic breast cancer: the Breast Cancer Clinical Outcome Measures (BCCOM) Project. $\mathrm{Br} J$ Cancer 2009;101:395-402

36 Holman LL, Friedman S, Daniels MS, Sun CC, Lu KH. Acceptability of prophylactic salpingectomy with delayed oophorectomy as riskreducing surgery among BRCA mutation carriers. Gynecol Oncol 2014;133:283-6.

37 Arts-de Jong $M$, Harmsen MG, Hoogerbrugge N, Massuger LF, Hermens RP, de Hullu JA. Risk-reducing salpingectomy with delayed oophorectomy in BRCA1/2 mutation carriers: patients' and professionals' perspectives. Gynecol Oncol 2015;136:305-10.

38 O'Connor AM. Validation of a decisional conflict scale. Med Decis Making 1995;15:25-30.

39 O'Connor AM. User Manual - Decision Regret Scale [document on internet]. Modified 2003 edn. Ottawa: Ottawa Hospital Research Institute; 1996. p. 3.

40 Gaba F, Piek J, Menon U, Manchanda R. Risk-reducing early salpingectomy and delayed oophorectomy as a two-staged alternative for primary prevention of ovarian cancer in women at increased risk: a commentary. BJOG 2019;126:831-9.

41 Beattie MS, Crawford B, Lin F, Vittinghoff E, Ziegler J. Uptake, time course, and predictors of risk-reducing surgeries in BRCA carriers. Genet Test Mol Biomarkers 2009;13:51-6.

42 Botkin JR, Smith KR, Croyle RT, Baty BJ, Wylie JE, Dutson D, et al. Genetic testing for a BRCA1 mutation: prophylactic surgery and screening behavior in women 2 years post testing. Am J Med Genet Part A 2003;118a:201-9
43 Bradbury AR, Ibe CN, Dignam JJ, Cummings SA, Verp $M$, White $M A$, et al. Uptake and timing of bilateral prophylactic salpingooophorectomy among BRCA1 and BRCA2 mutation carriers. Genet Med 2008;10:161-6.

44 Chai $X$, Friebel TM, Singer CF, Evans DG, Lynch HT, Isaacs $C$, et al. Use of risk-reducing surgeries in a prospective cohort of 1,499 BRCA1 and BRCA2 mutation carriers. Breast Cancer Res Treat 2014; 148:397-406

45 Cragun D, Weidner A, Lewis C, Bonner D, Kim J, Vadaparampil ST, et al. Racial disparities in BRCA testing and cancer risk management across a population-based sample of young breast cancer survivors. Cancer 2017;123:2497-505.

46 D'Alonzo M, Pecchio S, Liberale V, Modaffari P, Biglia N, Piva E, et al. Satisfaction and impact on quality of life of clinical and instrumental surveillance and prophylactic surgery in BRCA-mutation carriers. Clin Breast Cancer 2018;18:e1361-e1366.

47 Evans DGR, Lalloo F, Shenton A, Clancy T, Hopwood P, Ashcroft L, et al. Uptake of risk-reducing surgery in unaffected women at high risk of breast and ovarian cancer is risk, age, and time dependent. Cancer Epidemiol Biomarkers Prev 2009;18:2318-24.

48 Finkelman BS, Rubinstein WS, Friedman S, Friebel TM, Dubitsky S, Schonberger NS, et al. Breast and ovarian cancer risk and risk reduction in Jewish BRCA1/2 mutation carriers. J Clin Oncol 2012;30:1321-8

49 Flippo-Morton T, Walsh K, Sarantou T, White RL, Chambers K, Amacker-North $L$, et al. Surgical decision making in the BRCApositive population: institutional experience and comparison with recent literature. Breast J 2016;22:35-44

50 Friebel TM, Domchek SM, Neuhausen SL, Wagner T, Evans DG, Isaacs C, et al. Bilateral prophylactic oophorectomy and bilateral prophylactic mastectomy in a prospective cohort of unaffected BRCA1 and BRCA2 mutation carriers. Clin Breast Cancer 2007;7:875-82.

51 Kim SI, Lim MC, Lee DO, Seo SS, Kang S, Park SY, et al. Uptake of risk-reducing salpingo-oophorectomy among female BRCA mutation carriers: experience at the National Cancer Center of Korea. J Cancer Res Clin Oncol 2016;142:333-40.

52 Kram V, Peretz T, Sagi M. Acceptance of preventive surgeries by Israeli women who had undergone BRCA testing. Fam Cancer 2006;5:327-35.

53 Kwong A, Wong $\mathrm{CH}$, Shea C, Suen DT, Choi CL. Choice of management of southern Chinese BRCA mutation carriers. World J Surg 2010;34:1416-26

54 Lerman C, Hughes C, Croyle RT, Main D, Durham C, Snyder C, et al. Prophylactic surgery decisions and surveillance practices one year following BRCA1/2 testing. Prev Med 2000:31:75-80.

55 Lodder LN, Frets PG, Trijsburg RW, Meijers-Heijboer EJ, Klijn JGM, Seynaeve $C$, et al. One year follow-up of women opting for presymptomatic testing for BRCA1 and BRCA2: emotional impact of the test outcome and decisions on risk management (Surveillance or Prophylactic Surgery). Breast Cancer Res Treat 2002;73:97-112.

56 Mai PL, Piedmonte M, Han PK, Moser RP, Walker JL, Rodriguez $G$, et al. Factors associated with deciding between riskreducing salpingo-oophorectomy and ovarian cancer screening among high risk women enrolled in GOG-0199: An NRG Oncology/Gynecologic Oncology Group study. Gynecol Oncol 2017:145:122-9

57 Manchanda R, Burnell M, Johnson M, Sharma A, Gessler S, Side L, et al. Factors influencing uptake and timing of risk reducing salpingo- oophorectomy in women at risk of familial ovarian cancer: A competing risk time to event analysis. BJOG 2012;119:527-36. 
58 Meijers-Heijboer EJ, Verhoog LC, Brekelmans CT, Seynaeve C, Tilanus-Linthorst MM, Wagner A, et al. Presymptomatic DNA testing and prophylactic surgery in families with a BRCA1 or BRCA2 mutation. Lancet 2000;355:2015-20.

59 Metcalfe KA, Ghadirian P, Rosen B, Foulkes W, Kim-Sing C, Eisen A, et al. Variation in rates of uptake of preventive options by Canadian women carrying the BRCA1 or BRCA2 genetic mutation. Open Med 2007; 1:e92-e98.

60 Metcalfe KA, Liede A, Hoodfar E, Scott A, Foulkes WD, Narod SA An evaluation of needs of female BRCA1 and BRCA2 carriers undergoing genetic counselling. J Med Genet 2000;37:866-74.

61 Nebgen DR, Hurteau J, Holman LL, Bradford A, Munsell MF, Soletsky $B R$, et al. Bilateral salpingectomy with delayed oophorectomy for ovarian cancer risk reduction: A pilot study in women with BRCA1/2 mutations. Gynecol Oncol 2018;150:79-84.

62 Pezaro C, James P, McKinley J, Shanahan M, Young MA, Mitchell $G$. The consequences of risk reducing salpingo-oophorectomy: the case for a coordinated approach to long-term follow up post surgical menopause. Fam Cancer 2012;11:403-10.

63 Ray JA, Loescher LJ, Brewer M. Risk-reduction surgery decisions in high risk women seen for genetic counseling. J Genet Couns 2005;14:473-84

64 Schwartz MD, Isaacs C, Graves KD, Poggi E, Peshkin BN, Gell C, et al. Long-term outcomes of BRCA1/BRCA2 testing: risk reduction and surveillance. Cancer 2012;118:510-7.

65 Skytte AB, Gerdes AM, Andersen MK, Sunde L, Brondum-Nielsen K, Waldstrom $M$, et al. Risk-reducing mastectomy and salpingooophorectomy in unaffected BRCA mutation carriers: uptake and timing. Clin Genet 2010;77:342-9.

66 Tiller K, Meiser B, Butow P, Clifton M, Thewes B, Friedlander M, et al. Psychological impact of prophylactic oophorectomy in women at increased risk of developing ovarian cancer: a prospective study. Gynecol Oncol 2002;86:212-9.

67 Uyei A, Peterson SK, Erlichman J, Broglio K, Yekell S, Schmeler K, et al. Association between clinical characteristics and risk-reduction interventions in women who underwent BRCA1 and BRCA2 testing: a single-institution study. Cancer 2006;107:2745-51.

68 Westin SN, Sun CC, Lu KH, Schmeler KM, Soliman PT, Lacour RA et al. Satisfaction with ovarian carcinoma risk-reduction strategies among women at high risk for breast and ovarian carcinoma. Cancer 2011;117:2659-67.

69 Garcia C, Wendt J, Lyon L, Jones J, Littell RD, Armstrong MA, et al. Risk management options elected by women after testing positive for a BRCA mutation. Gynecol Oncol 2014;132:428-33.

70 Schmeler KM, Sun CC, Bodurka DC, White KG, Soliman PT, Uyei $A R$, et al. Prophylactic bilateral salpingo-oophorectomy compared with surveillance in women with BRCA mutations. Obstet Gynecol 2006 108(3 Pt 1):515-20.
71 Babb SA, Swisher EM, Heller HN, Whelan AJ, Mutch DG, Herzog TJ, et al. Qualitative evaluation of medical information processing needs of 60 women choosing ovarian cancer surveillance or prophylactic oophorectomy. J Genet Couns 2002;11:81-96.

72 Finch A, Metcalfe KA, Chiang JK, Elit L, McLaughlin J, Springate C, et al. The impact of prophylactic salpingo-oophorectomy on menopausal symptoms and sexual function in women who carry a BRCA mutation. Gynecol Oncol 2011;121:163-8.

73 Cohen JV, Chiel L, Boghossian L, Jones M, Stopfer JE, Powers J, et al. Non-cancer endpoints in BRCA1/2 carriers after risk-reducing salpingo-oophorectomy. Fam Cancer 2012;11:69-75.

74 Vermeulen RFM, Mv B, Kieffer JM, Bleiker EMA, Valdimarsdottir $H B$, Massuger LFAG, et al. Hormone replacement therapy after risk-reducing salpingo-oophorectomy minimises endocrine and sexual problems: A prospective study. Eur J Cancer 2017;84:15967.

75 Tucker PE, Bulsara MK, Salfinger SG, Tan JJ, Green H, Cohen PA. The effects of pre-operative menopausal status and hormone replacement therapy (HRT) on sexuality and quality of life after riskreducing salpingo-oophorectomy. Maturitas 2016;85:42-8.

76 Hallowell N. A qualitative study of the information needs of high risk women undergoing prophylactic oophorectomy. PsychoOncology 2000;9:486-95.

77 Gaba F, Manchanda R. Systematic review of acceptability, cardiovascular, neurological, bone health and HRT outcomes following risk reducing surgery in BRCA carriers. Best Pract Res Clin Obstet Gynaecol 2020;65:46-65.

78 Rebbeck TR, Friebel T, Wagner T, Lynch HT, Garber JE, Daly MB, et al. Effect of short-term hormone replacement therapy on breast cancer risk reduction after bilateral prophylactic oophorectomy in BRCA1 and BRCA2 mutation carriers: the PROSE Study Group. J Clin Oncol 2005:23:7804-10.

79 Kotsopoulos J, Huzarski T, Gronwald J, Moller P, Lynch HT, Neuhausen SL, et al. Hormone replacement therapy after menopause and risk of breast cancer in BRCA1 mutation carriers: a case-control study. Breast Cancer Res Treat 2016;155:365-73.

80 Eisen A, Lubinski J, Gronwald J, Moller P, Lynch HT, Klijn J, et al. Hormone therapy and the risk of breast cancer in BRCA1 mutation carriers. J Natl Cancer Inst 2008;100:1361-7.

81 Challberg J, Ashcroft L, Lalloo F, Eckersley B, Clayton R, Hopwood $P$, et al. Menopausal symptoms and bone health in women undertaking risk reducing bilateral salpingo-oophorectomy: significant bone health issues in those not taking HRT. Br J Cancer 2011;105:22-7.

82 Finch A, Metcalfe KA, Chiang J, Elit L, McLaughlin J, Springate C, et al. The impact of prophylactic salpingo-oophorectomy on quality of life and psychological distress in women with a BRCA mutation. Psycho-Oncology 2013;22:212-9. 